\title{
Digital coherent receiver for orbital angular momentum demultiplexing
}

\author{
Aniceto Belmonte ${ }^{1, *}$ and Juan P. Torres ${ }^{1,2}$ \\ ${ }^{1}$ Technical University of Catalonia, BarcelonaTech, Department of Signal Theory and Communications, Barcelona 08034, Spain \\ ${ }^{2}$ ICFO_Institut de Ciencies Fotoniques, Mediterranean Technology Park, Castelldefels, Barcelona 08860, Spain \\ ${ }^{*}$ Corresponding author: belmonte@tsc.upc.edu
}

Received November 15, 2012; revised December 19, 2012; accepted December 19, 2012;

posted December 20, 2012 (Doc. ID 180044); published January 15, 2013

\begin{abstract}
We put forward a type of receiver for coherent detection of the photon orbital angular momentum (OAM). A coherent array receiver, consisting of multiple subapertures, with each subaperture coupled to a single-mode fiber, maps the complex optical field in the image plane. Using digital samplers connected to each array element, the local electrical signals resulting from the detection process can be measured coherently, moving the complexity of the full OAM measurement from the optical domain to the digital domain. By computer processing the coherent electrical patterns obtained, one can retrieve full information (amplitude and phase) of the different OAM components that constitute any incoming beam. (c) 2013 Optical Society of America

OCIS codes: $\quad 050.4865,140.3295,060.4230$.
\end{abstract}

Most photonic applications make use of the frequency or the polarization of light to encode or retrieve information. Notwithstanding, the spatial shape of light also constitutes a degree of freedom that can be used for the same purpose, and indeed, it is increasingly being used in new photonic applications [1]. For instance, one can encode information simultaneously on a few collinear beams that otherwise have the same wavelength or polarization, but are on a separate channel because their spatial shapes, or modes, are orthogonal [2], effectively increasing the spectral efficiencies of free-space optical links. In imaging and probing systems, one can illuminate an unknown target with a convenient spatial shape, and measure the energy carried by some selected modes of the reflected light to obtain information about the target [3].

The spatial shape of a light beam is conveniently described in terms of modes that bear orbital angular momentum (OAM), modes which show a characteristic azimuthal phase dependence of the form $\exp (j m \phi)$. Even though the general considerations discussed in this Letter will apply to both Bessel beams [4] and LaguerreGaussian beams []ㅡ as well, for the sake of simplicity, we will mostly refer to the last case from now on.

Light beams with arbitrary spatial shapes will show correspondingly specific OAM mode decompositions or spiral spectrum. Currently available technology offers different possibilities to detect OAM modes. Appropriately designed spiral phase plates []], and q-plates [] ] can be used to produce the required phase distribution for detecting a vortex beam. Spatial light modulators and computer-generated holograms have played an outstanding role $[8,9]$. Some of these schemes can be used to obtain partial information about the spiral spectrum, for instance, the amplitude of the OAM modes [10], but they are generally insensitive to the phase of the modes. This turns out to be an important drawback for systems that encode amplitude and phase information in OAM modes, as is the case in certain modulation schemes [2].

In this Letter, we describe a digital technique that can retrieve the whole spiral spectrum (amplitude and phase) of a spatial light beam capturing a portion of the incoming beam. The idea is to perform a coherent detection of the captured field. With a coherent system, we have access to all the degrees of freedom of the complex electromagnetic field, including amplitude, phase, and polarization. A coherent receiver measures these degrees of freedom by making the received optical signal interfere with a reference optical signal or local oscillator. Coherent receivers enable baseband detection and generate an electrical signal at intermediate radio frequencies. Recent advances in fiber coherent optical communication systems have stimulated interest in applying coherent detection in other laser systems $[11,12]$.

Figure 1 shows a sketch of the configuration proposed. A spherical lens plays the part of light collector. At the focal plane of the lens, a circular array of coherent receivers maps the entire optical field into the digital domain. Using digital samplers connected directly to each element of the array, the local, instantaneous electrical signal vectors can be measured coherently up to the gigahertz range, enabling their manipulation and processing entirely in software. This is one of the main motivations for using coherent detection, as the complexity of the OAM measurement moves from the optical domain to the digital domain.

For computing the OAM spectrum, we make use of a simple discrete Fourier transform (DFT), which enables

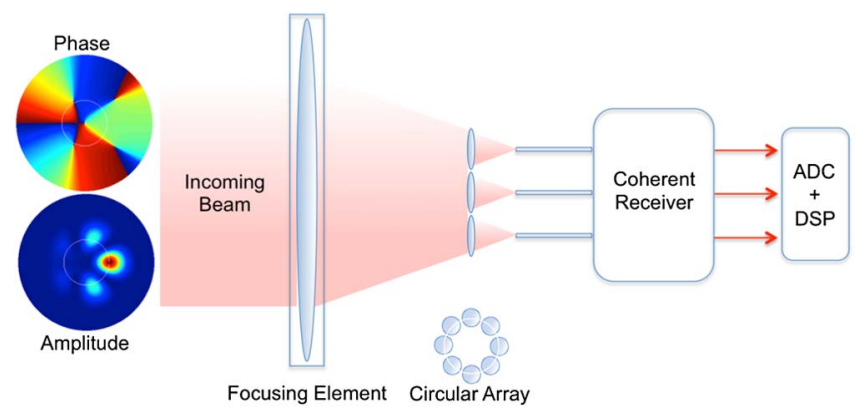

Fig. 1. (Color online) Sketch of the OAM demultiplexer. At the focal plane of a focusing system, a circular array of coherent receivers is used to map the entire optical field into the digital domain. An analog-to-digital converter (ADC) is used in conjunction with digital signal processing (DSP) manipulation. 
equalization of possible linear impairments in the receiving process. The Fourier transform maps continuum functions (such as optical fields carrying OAM) into discrete sequences and permits rapid numerical calculation of the harmonic components of the function. Such discrete analysis is well matched to digital processing competence.

Let us assume that the complex amplitude $U$ of a monochromatic light beam of frequency $f$ is formed by a linear combination of a finite set of OAM modes

$$
U(\rho, \phi, t)=\sum_{m} C_{m} U_{m}(\rho, \phi) \exp (j 2 \pi f t),
$$

where $(\rho, \phi)$ are polar coordinates and $C_{m}$ are complex amplitudes, $m=0, \pm 1, \pm 2, \ldots, \pm M$, and $M$ is the order of the highest harmonic mode present in the light beam. The OAM modes, which define an orthogonal basis, read as

$$
U_{m}(\rho, \phi)=R_{m}(\rho) \exp (-j m \phi),
$$

where $m$ is the OAM mode number, and the azimuthal term $m \phi$ describes the phase dependence in a plane perpendicular to the beam axis. This means that for any $m \neq 0$, the phase fronts of modes are not planar but helical. Each integer $m$ characterizes a different OAM state of the helical beam. In Eq. (2), $R_{m}(\rho)$ defines the radial amplitudes. The phase configuration of the OAMgenerating system gives rise to coherent OAM-carrying optical beams with characteristic on-axis phase singularities and hence amplitude nulls at the centers of the beams. Both Bessel beams as well as Laguerre-Gaussian beams are described by Eq. (2).

The spherical lens acts as a light collector and plays the part of a light field spatial analyzer. An ideal spherical lens of aperture diameter $D$ and focal length $f$ performs the Fourier transform $E(v, \theta)=\mathfrak{I}\lceil U(\rho, \phi)\rceil$, where

$$
E(v, \theta, t)=\sum_{m} C_{m} E_{m}(v) \exp (j m \theta) \exp (j 2 \pi f t) .
$$

$E_{m}(v)$ and $R_{m}(\rho)$ are related through the Hankel transform of the $m$ th order,

$$
E_{m}(v)=2 \pi \frac{k}{f}(-j)^{m} \int_{0}^{D / 2} \rho d \rho R_{m}(\rho) J_{m}\left(\frac{k}{f} \rho v\right) .
$$

Here, $J_{m}(\cdot)$ is the $m$-order Bessel function of the first kind.

A circular array of apertures located at the focal plane of the sampling lens, connected to a corresponding array of single-mode fibers (see Fig. 1), is used to sample the incoming beam. The array elements are distributed equidistantly around the perimeter of a circle. Careful fiber layout ensures that they have identical optical path lengths. Also, the truncation parameter $\tau=d / 2 \omega_{m}$, describing the coupling of the fiber to the incoming signal, must be chosen properly to maximize the receiving power. Here, $d$ is the sampling aperture diameter and $\omega_{m}$ is the fiber's mode radius. A value $\tau=1.12$ is optimal when the incident wave is fully coherent [12]. The same value of the truncation parameter $\tau$ is used for each subaperture fiber. After coherent detection and filtering, to remove the carrier frequency $f$ and its harmonics, the signal produced by each element of the array writes

$$
I_{q}=\eta \sum_{m} C_{m} E_{m}\left(v_{0}\right) \exp \left(j m \theta_{q}\right)
$$

where $q=1,2, \ldots, N, N$ being the number of apertures in the circular array. The azimuthal angles $\theta_{q}$ indicate the angular position of each element of the array along the circle of radius $v_{0}$. Here, $\eta$ describes the efficiency of the coherent detection process and, without lost of generality, can be considered equal to unity.

The sampling (Nyquist) theorem shows that the original optical signal can be perfectly reconstructed from this finite sequence of samples if the number of apertures $N$ in the circular arrangement exceeds $2 M$, where $M$ is the highest angular frequency of the original signal. The largest $M$ mode the optical system can measure $|M|<$ $N / 2$ is determined then by the number of elements of the array. Certainly, increasing the value of $N$ of the array, and sampling the optical signal with a spatial frequency significantly higher, may help in avoiding aliasing and reducing noise. However, the maximum number of array elements $N$ is also limited by the achievable signalto-noise ratio.

For computing the OAM spectrum, we make use of a DFT. The sampling of the incoming field performed by the array creates the discrete inputs to the DFT. By computer processing the coherent electrical patterns given in Eq. (5), we can retrieve all OAM components, its amplitude, and its phase. The complex current $I(v, \theta)$ can always be expanded into the functional Fourier series in terms of angular harmonics by using a DFT

$$
I_{q}=\sum_{q} i_{q} \exp (j q \theta)
$$

where

$$
i_{q}=\frac{1}{2 \pi} \int_{0}^{2 \pi} I_{q} \exp (-j q \theta)
$$

Then, by replacing Eq. (5) in Eq. (7), and noticing that the subsequent angular integral is $2 \pi$ when $q=m$ and zero otherwise, it yields

$$
i_{q}=\sum_{m} C_{m} E_{m}\left(v_{0}\right) \delta(q-m)
$$

with $\delta(\cdot)$ being the Dirac delta function. Comparing Eqs. (ㅁ) and (8), we obtain

$$
C_{m}=\frac{i_{m}}{E_{m}\left(v_{0}\right)} .
$$

The term $E_{m}\left(v_{0}\right)$ is a known constant for every coefficient $C_{m}$, as described in Eq. (4). Interestingly, the terms $E_{m}\left(v_{0}\right)$ can be calibrated for any specific experimental setup by feeding single OAM eigenstates with mode 
(a)

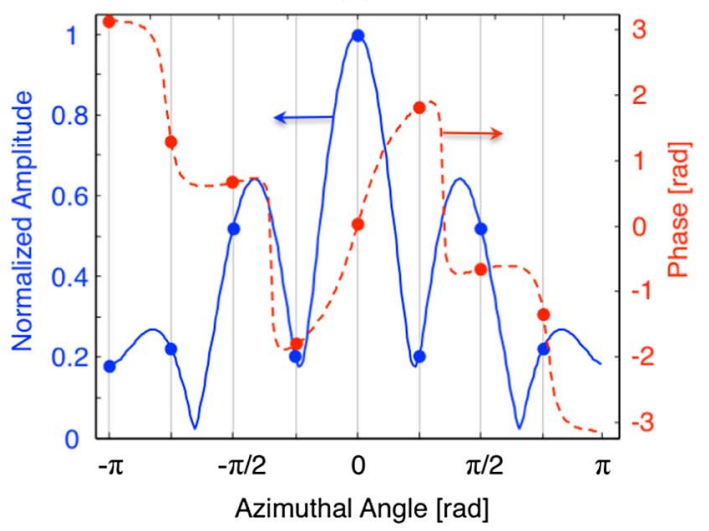

(b)

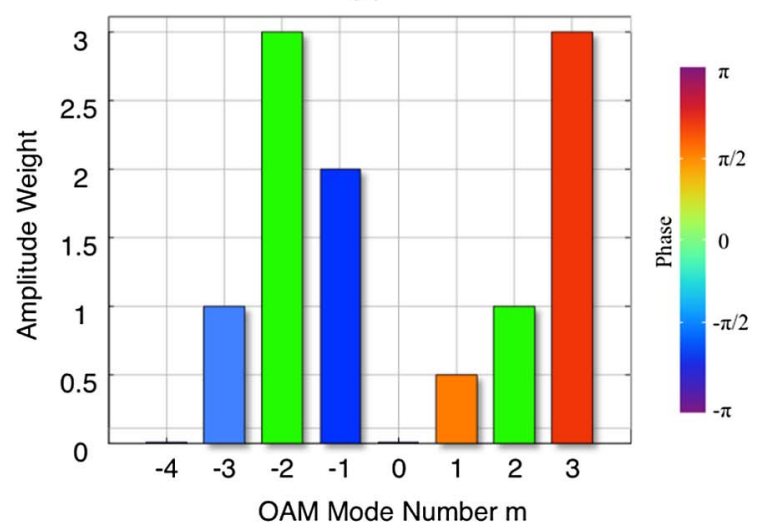

Fig. 2. (Color online) Coherent array receiver maps the complex optical field in the image plane. In (a), using digital samplers connected to each array element, the local, instantaneous electrical signals resulting from the detection process can be measured coherently. In (b), by computer processing the coherent electrical patterns obtained, one can retrieve full information (amplitude and phase) of the different OAM components that constitute the incoming beam [it plots the phases in a continuous color (gray) scale and the amplitude as bar lengths].

number $m$ at the input of the analyzer. In this case, $C_{m}=$ 1 and the term is directly measured as $E_{m}\left(v_{0}\right)=i_{m}$.

The proposed OAM demultiplexing technique is sensitive to tilt aberration in the receiving optics. Tilt, described in terms of $\rho \cos \phi$ and $\rho \sin \phi$ depends on the azimuthal angle coordinate $\phi$, and it could distort the OAM decomposition of the light reaching the coherent receiver. If present, tilt may affect the OAM measurements, and a carefully alignment of the multiplexing array with respect to the axis of the incoming light beam may be required. Interestingly, because defocus aberration does not depend on the azimuthal angle $\phi$, it only depends on the radial coordinate $\rho$ through $\left(\rho^{2}-1\right)$; the effect of defocus can be ignored. A relevant presence of other phase aberrations, such as astigmatism and coma, generally decreases as the aberration order increases.

Figure 2(a) shows the complex electrical signal detected when a circular array of $N=8$ elements maps a complex optical field consisting of $M=7$ OAM states, from $m=-3$ to $m=3$. Solid curves represent the field amplitude (blue), while dashed curves represent the phase (red) at the perimeter of a circle containing the array. The array samples the field amplitude and phase at specific azimuthal angles, as indicated by the dots and vertical curves in the plot.

The use of coherent systems benefit the work developed in the areas of coherent optical communications and signal processing techniques, where these coherent methods are routinely used. In Fig. 2(b), the spiral spectral content (amplitude and phase) of the complex light beam captured by the circular array is completely retrieved by the digital analysis applied to the coherent signals produced by the array coherent receivers. The sampling of the electrical signals performed by the array creates the discrete inputs to the DFT used for computing the OAM spectrum. Figure 2(b) shows both the amplitude and the phase of all OAM components retrieved by the technique.

In conclusion, this Letter has discussed the capabilities of using a circular array of coherent receivers as an OAM digital multiplexer to retrieve the whole spiral spectrum (amplitude and phase) of an incoming complex optical beam. Using digital sampling and processing techniques, the complexity of the optical field moves to the digital domain and enables the measurement of the information carried out by the different OAM components coherently. Based on Shannon's law, the highest data rate supported in each OAM channel is only limited by its bandwidth and the achievable signal-to-noise ratio. The Nyquist theorem explains the relationship between the digital sampling rate and the available channel bandwidth. Spectrally efficient modulation formats, along with the benefits afforded by digital coherent receivers, allow for the implementation of OAM channels supporting multi-Gbps data rates $[\underline{11}, 12]$.

This work was supported by the Government of Spain (TEC2009-10025, TEC2012-34799, and FIS2010-14831), by PHORBITECH (grant 255914), and by Fundacio Privada Cellex Barcelona.

\section{References}

1. J. P. Torres and L. Torner, Twisted Photons: Applications of Light with Orbital Angular Momentum (Wiley, 2011).

2. J. Wang, J. Y. Yang, I. M. Fazal, N. Ahmed, Y. Yan, H. Huang, Y. Ren, Y. Yue, S. Dolinar, M. Turand, and A. E. Willner, Nat. Photonics 6, 488 (2012).

3. L. Torner, J. P. Torres, and S. Carrasco, Opt. Express 13, 873 (2005).

4. J. Durnin, J. Opt. Soc. Am. A 4, 651 (1987).

5. L. Allen, M. W. Beijersbergen, R. J. C. Spreeuw, and J. P. Woerdman, Phys. Rev. A 45, 8185 (1992).

6. M. W. Beijersbergen, R. P. C. Coerwinkel, M. Kristensen, and J. P. Woerdman, Opt. Commun. 112, 321 (1994).

7. L. Marrucci, C. Manzo, and D. Paparo, Phys. Rev. Lett. 96, 163905 (2006)

8. V. Y. Bazhenov, M. V. Vasnetsov, and M. S. Soskin, JETP Lett. 52, 429 (1990).

9. N. R. Heckenberg, R. McDuff, C. P. Smith, and A. G. White, Opt. Lett. 17, 221 (1992).

10. G. C. G. Berkhout, M. P. J. Lavery, J. Courtial, M. W. Beijersbergen, and M. J. Padgett, Phys. Rev. Lett. 105, 153601 (2010).

11. G. Li, Adv. Opt. Photon. 1, 279 (2009).

12. A. Belmonte and J. M. Kahn, Opt. Express 18, 3928 (2010). 